\title{
Research on Modeling Method of 3D Geological Entity Model Based on BIM
}

\author{
Huang Si-jie ${ }^{1,2,3}$, Guo Yu-bin ${ }^{1,2,3}$, Yu Chang-yi ${ }^{1,2,3}$, Wang Xiao ${ }^{4 *}$ and Wang Qi-zhi ${ }^{4}$ \\ ${ }^{1}$ CCCC Tianjin Port Engineering Institute Co., Ltd., 300222 Tianjin, China \\ ${ }^{2}$ Key Laboratory of Port Geotechnical Engineering of the Ministry of Communication, 300222 Tianjin, China \\ ${ }^{3}$ Key Laboratory of Port Geotechnical Engineering of Tianjin, 300222 Tianjin, China \\ ${ }^{4}$ Hebei University of Science and Technology, 050018 Shijiazhuang, China
}

\begin{abstract}
The paper uses the BIM design platform Revit as the main program for modeling and debugging, relying on Lizheng survey drilling data, based on the Revit API secondary development technology and its modeling method, to carry out various algorithms involved in 3D geological modeling . The program design realizes automation and rapid establishment of 3D geological entity model, and enables it to have more comprehensive geological information query, section analysis and other functions. At the same time, in order to solve the problems of insufficient original borehole data or uneven borehole distribution, the paper proposes a virtual borehole generation method based on the moving quadric surface fitting interpolation algorithm, which effectively improves the modeling accuracy and the visualization effect of the model.
\end{abstract}

\section{Preface}

Drilling data plays a vital role in 3D stratum simulation due to its intuitive, accurate and detailed characteristics. Constructing a 3D stratum solid model based on the drilling data can be used for engineering design compared with traditional 2D drill profiles. It provides people with more intuitive and effective analysis and decision-making tools. In terms of 3D geological modeling, Lin Bingxian and others proposed three virtual drilling concepts for different stages of 3D geological body modeling, and based on this, designed a virtual drilling control 3D geological body model construction method ${ }^{[1]}$; based on the Revit environment, Cai Guodong and others explored the application of BIM technology in the rapid modeling of 3D stratum information models ${ }^{[2]}$; Peng Jianlin used methods such as cubic spline interpolation to expand the borehole data, and based on this, drew the engineering 3D geological model ${ }^{[3]}$; $\mathrm{Chu}$ Shili and others summarized the advantages and disadvantages of commonly used BIM software to create geological models, and proposed a method to quickly create geological models by combining Civil $3 \mathrm{D}$ and Revit ${ }^{[4]}$.

Based on the above research, it is found that the three-dimensional geological model controlled by drilling data still has problems such as poor model accuracy, low modeling efficiency, and lack of stratum information. Revit is a widely used three-dimensional modeling platform with its powerful visualization, information, and the advantages of parameterization provide an effective solution for the rapid establishment of 3D geological solid models. Based on the secondary development technology of Revit and the drilling data of Lizheng, the paper proposes a method to quickly generate a three-dimensional geological solid model, and completes the development of virtual interpolation drilling and geological body section analysis plug-ins, which has the meaning of strong reference in geotechnical engineering survey.

\section{Generate virtual borehole}

The scientific and effective spatial interpolation algorithm can solve the problems of uneven borehole distribution and insufficient data, and meet the needs of actual BIM modeling. In this paper, the mobile quadratic surface fitting algorithm is used to interpolate the drilling data, and the algorithm process is written as an application plug-in, which is convenient for engineering designers to process the drilling data.

\subsection{Implement interpolation algorithm}

The moving quadric surface fitting method takes the coordinates of the orifice to be inserted into the virtual borehole as the center origin, establishes the corresponding quadric surface function, and enters the search radius to obtain all DEM elevation point data in 
the circular area as the solution condition of the function. If the number of elevation points in the area is less than 6, the search radius is expanded to obtain the virtual borehole elevation. The calculation flow of this method is shown in Figure 1.

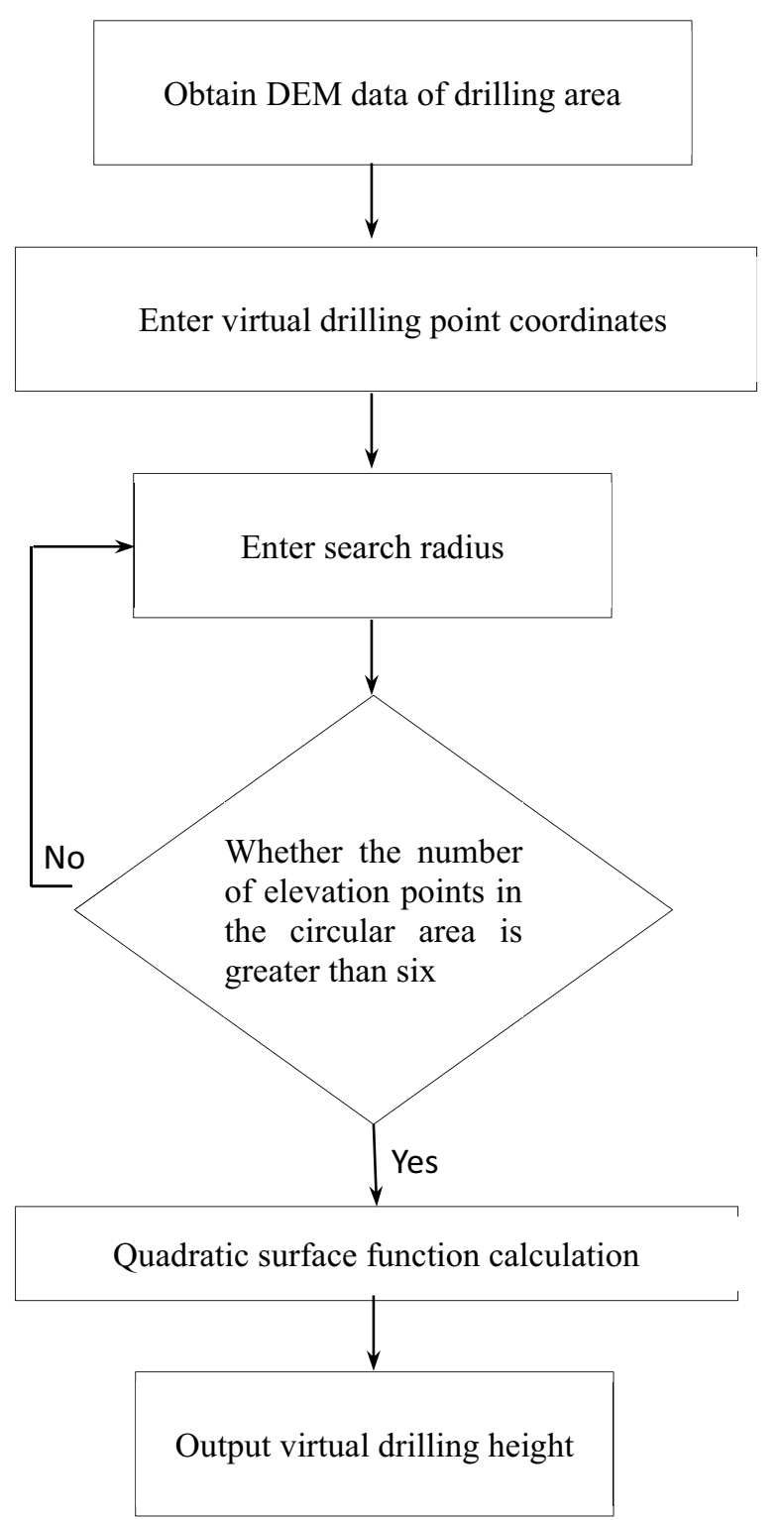

Fig. 1 Flow chart of interpolation algorithm for moving quadric surface fitting

The application of this method is more flexible, the algorithm is simple, the calculation time is short, and the calculation accuracy is high. After determining the two-dimensional coordinates of the point to be inserted, the formula (1) is selected as the fitting surface, and the corresponding error equation is the formula (2).

$$
\begin{aligned}
& \mathrm{z}=A x^{2}+B x y+C y^{2}+E y+F \\
& v_{i}=A \bar{X}_{i}^{2}+B \bar{X}_{i} \bar{Y}_{i}+C \bar{Y}_{i}^{2}+D \bar{X}_{i}+E \bar{Y}_{i}+F
\end{aligned}
$$

The error equation $V=M X-Z$ is obtained from $\mathrm{n}$ data points greater than 6 in the search area, where:

$$
\begin{aligned}
& \mathrm{Z}=\left[\begin{array}{c}
\mathrm{z}_{1} \\
\mathrm{z}_{1} \\
\vdots \\
\mathrm{z}_{n}
\end{array}\right] \quad \mathrm{V}=\left[\begin{array}{c}
\mathrm{v}_{1} \\
\mathrm{v}_{1} \\
\vdots \\
\mathrm{v}_{n}
\end{array}\right] \\
& \mathrm{M}=\left[\begin{array}{cccccc}
\bar{X}_{1}^{2} & \bar{X}_{1} \bar{Y}_{1} & \bar{Y}_{1}^{2} & \bar{X}_{1} & \bar{Y}_{1} & 1 \\
\bar{X}_{2}^{2} & \bar{X}_{2} \bar{Y}_{2} & \bar{Y}_{2}^{2} & \bar{X}_{2} & \bar{Y}_{2} & 1 \\
\vdots & \vdots & \vdots & \vdots & \vdots & \vdots \\
\bar{X}_{n}^{2} & \bar{X}_{n} \bar{Y}_{\mathrm{n}} & \bar{Y}_{n}{ }^{2} & \bar{X}_{n} & \bar{Y}_{n} & 1
\end{array}\right]
\end{aligned}
$$

$$
\mathrm{X}=\left[\begin{array}{lllll}
A & B & C & \cdots & F
\end{array}\right]^{T}
$$

Calculate the weight of each data point. The weight Pi here does not represent the observation accuracy of the point, but reflects the spatial correlation of the point with the virtual borehole inserted. In this paper, the square of the distance is selected as the reciprocal weight According to adjustment theory ,the solution of moving quadratic surface coefficient is formula(5), Since the virtual drilling point is the coordinate origin, the parameter $\mathrm{F}$ is the orifice elevation value to be inserted into the virtual drilling.

$$
\mathrm{X}=\left(M^{T} P M\right)^{-1} M^{T} P Z
$$

\subsection{Drilling data processing}

The thesis completes the virtual borehole data processing plug-in based on the moving quadric surface interpolation algorithm. Its main functions are to generate a single virtual borehole, area virtual borehole, borehole drawing, borehole information and rock layer information output. The plug-in performs interpolation or extrapolation on the basis of rationalized borehole data. It can accurately insert a single virtual borehole by reading DEM data, rock formation data, borehole coordinate data and bad geological boundary data in the borehole area, or insert virtual holes evenly and continuously in the boundary area.

In order to increase the visualization effect of data processing, the plug-in developed a two-dimensional conceptual modeling function based on the CAD drawing theory, conceptually modeled the processed data, initially demonstrated the effect of generating data, and applied differently according to different formation information. The material maps help to distinguish different rock formations, and use simple two-dimensional drawings to increase the intuitive effect of design developers, which is conducive to understanding and further design and modification. Graphic drawing and formation data are shown in Figures 2 3. 


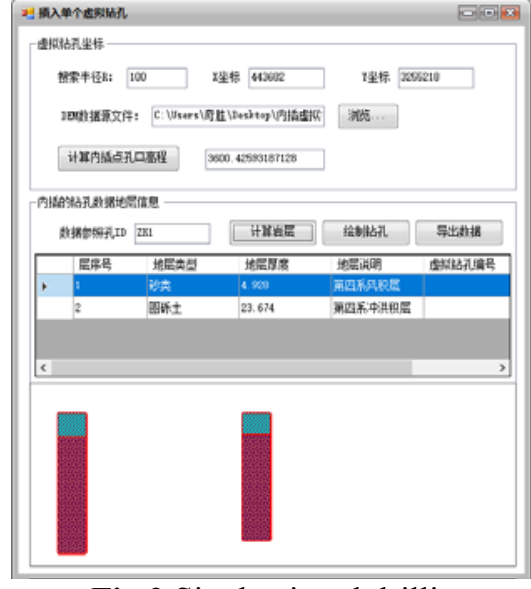

Fig.2 Single virtual drilling

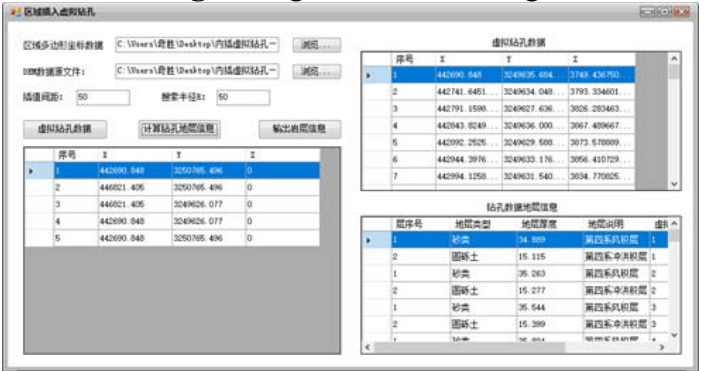

Fig. 3 Insert virtual drilling information in area

The drilling data processing plug-in solves the problem of insufficient drilling data and brings great help to the refined modeling of 3D geology. In order to facilitate the export of virtual borehole data, the plug-in developed an export module that can output borehole information and rock formation information into an Excel table in a fixed format. Using this form, the data can be edited again, and it is convenient to import into the Revit modeling platform to provide the necessary data processing support for $3 \mathrm{D}$ geological modeling. The example of exported data is shown in Figure 4.

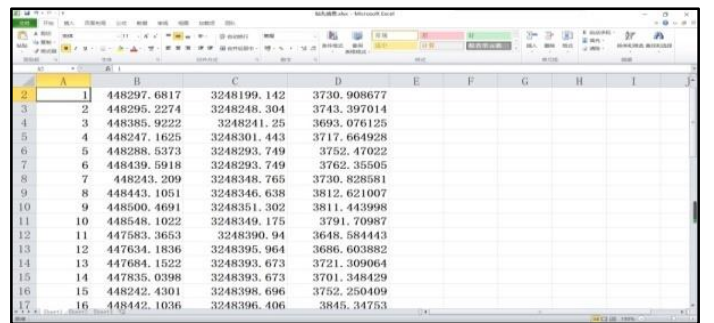

(a)Drilling information

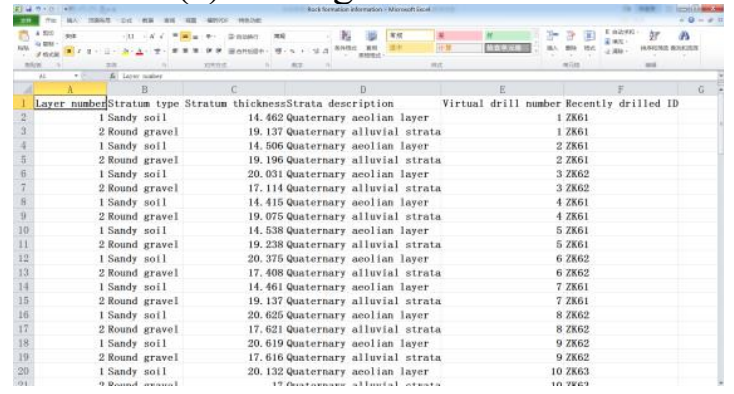

(b)Rock formation information

Fig. 4 Export data
In order to quickly create a three-dimensional geological entity model, the paper develops a geological modeling plug-in based on the Revit API. The main functions of the plug-in are to create a three-dimensional geological model, vertical excavation, and internal excavation. In the Revit operating environment, the plug-in reads the Excel geological drilling data in a specific format, automatically calculates the sequence of the missing geological layer, and uses different modeling methods to model the continuous formation and the missing formation to obtain the fastest Modeling speed .The established geological model is stored in a single layer in the form of Revit family files, and each layer of geological body can be individually edited for formation description information and material information.

\subsection{Formation data processing}

The borehole information contains the spatial information and geological layering information of the borehole. After data filtering, the borehole information used for 3D geological modeling needs to include the borehole number, plane coordinates, layer top elevation and other information. For complex geological conditions such as missing strata and thin out, in order to facilitate subsequent algorithm design, when constructing a three-dimensional geological entity model, the absolute elevation of the top and bottom of the rock layer needs to be calculated based on the borehole elevation and the thickness of the rock layer that passes through. Then set both the top and bottom values as the bottom layer of the previous layer, which is the hypothetical zero-thickness stratum ${ }^{[1]}$. According to the relationship between the absolute elevation of the roof and floor in the vertical direction of the borehole and the sequence law of the stratum, the spatial order relationship of the stratum can be determined, and then the stratum can be sorted ${ }^{[1]}$.

The processed formation data can clearly express complex and changeable formation information, unify the various drilling points with different formation sequences into the same structure, and solve the problem that it is difficult to express formation inversion and missing information in the 3D geological body model .Laid the foundation for the next step of structural reasoning and automatic modeling ${ }^{[1]}$.

\subsection{Create a model}

In order to quickly establish a three-dimensional geological model, the thesis uses separate modeling methods for continuous formations and complex formations separately, which greatly improves the modeling efficiency .The technical route is shown in Figure 5.

\section{D geological modeling}




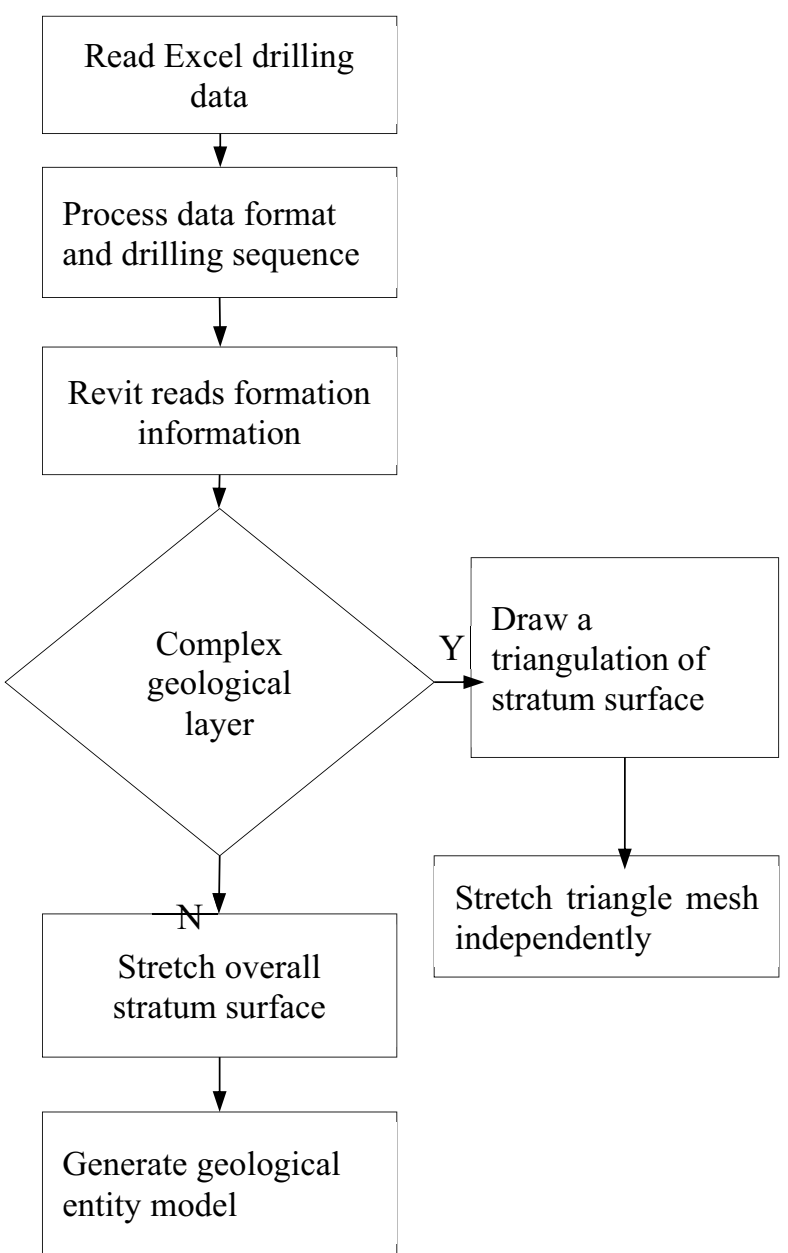

Fig.5 3D geological modeling technology roadmap

\subsubsection{Continuous formation modeling}

The drilling points of the continuous top and bottom of the stratum are only different in the Z-axis direction coordinates. The data of the drill points are stored in layers according to the stratigraphic sequence. In the Revit adaptive metric model, the local closed surface of the stratum roof is obtained by stripping the outer contour of the stratum. At the same time, the corresponding closed surface of the bottom plate is obtained, and the NewLoftForm method is used to stretch it into a local solid model, and finally spliced into a whole stratum solid model. The experimental data is 40 drill holes in 5 strata, and the creation of a single layer of continuous geological solid model takes only 40 seconds.

\subsubsection{Stratum model}

For complex formations, such as sharp geological conditions such as sharp breaks, folds, karst caves and faults, continuous formation modeling methods will not be able to generate local surfaces. To make modeling more flexible, you can draw irregular triangulation of formation surfaces and extract single triangular face down to stretch the body. For the missing area of the stratum, you can give up the stretching operation or generate a hollow shear solid model. This method requires a large amount of calculation. The modeling of a single-layer spiked geological body with 13 missing strata points takes 9 minutes. The physical effects and cross-sectional diagrams are shown in Figures 6 to 7 , where the yellow stratum is a sharp stratum.

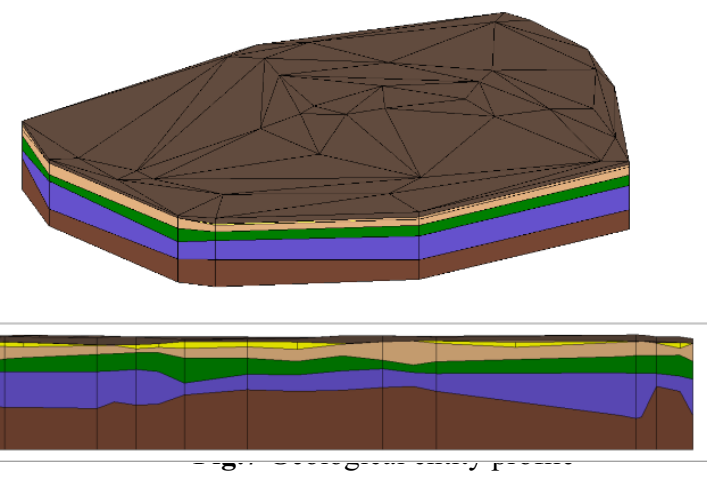

\subsection{Geological model editing}

The rapidly generated three-dimensional geological model can intuitively express the geological structure of the borehole area, but it shows less formation information. With the help of the geological modeling plug-in, the geological body can be cut simply and effectively, and the cutting path coordinates are output, to provide a data foundation for realizing 3D geological roaming in GIS platform.

\subsubsection{Vertical excavation}

Site survey and foundation pit construction have very high requirements for the gradual change of stratum inside the geological body. In order to observe the internal structure of the geological body in more detail, the geological body can be bored vertically. The vertical excavation function of the plug-in has three control parameters, namely the top elevation of the excavation, the bottom elevation and the excavation range. In the Revit operating environment, designers need to draw model lines on the basis of existing models, or import 3D coordinate point data as the boundary range of the excavation, and at the same time specify the upper and lower limits of the excavation, you can dig the geological body vertically Hole operation, the effect of digging holes is shown in Figure 8.

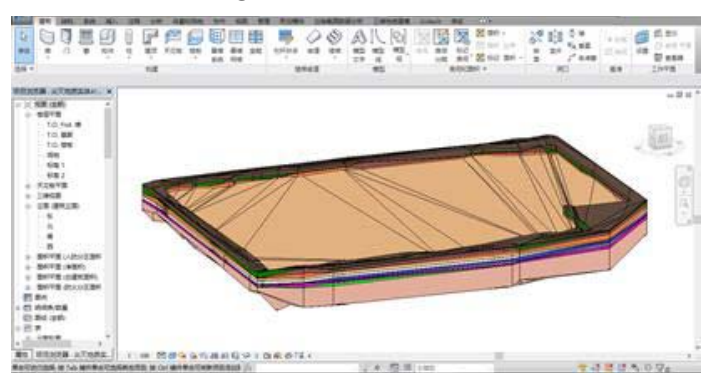

Fig.8 Excavation effect of elevation 


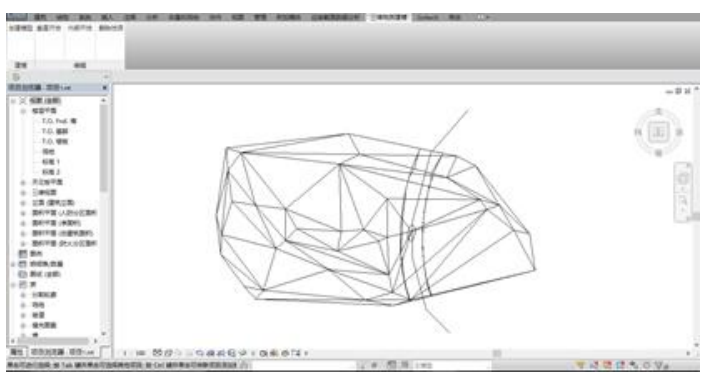

Fig. 9 Model line path plan 499 472m

\subsubsection{Internal excavation}

The application of the internal excavation function is more flexible, and the space shear volume can be created in the three-dimensional space according to the path direction .The designer needs to determine two parts in use: the path parameters, which can be determined by drawing the model line, or the path coordinate file can be imported; the cross-sectional profile parameters of the shear body: the program defaults to a round profile, and the profile diameter needs to be entered. Or you can achieve the digging operation of any shape contour by importing the local contour. The local contour needs to be created in the adaptive conventional model family. The model line path is selected as shown in Figure 9, and the excavation effect is shown in Figure 10 Figure 11. After the digging is completed, the path data and the BIM model are imported into the GIS platform to further achieve the effect of 3D geological roaming.
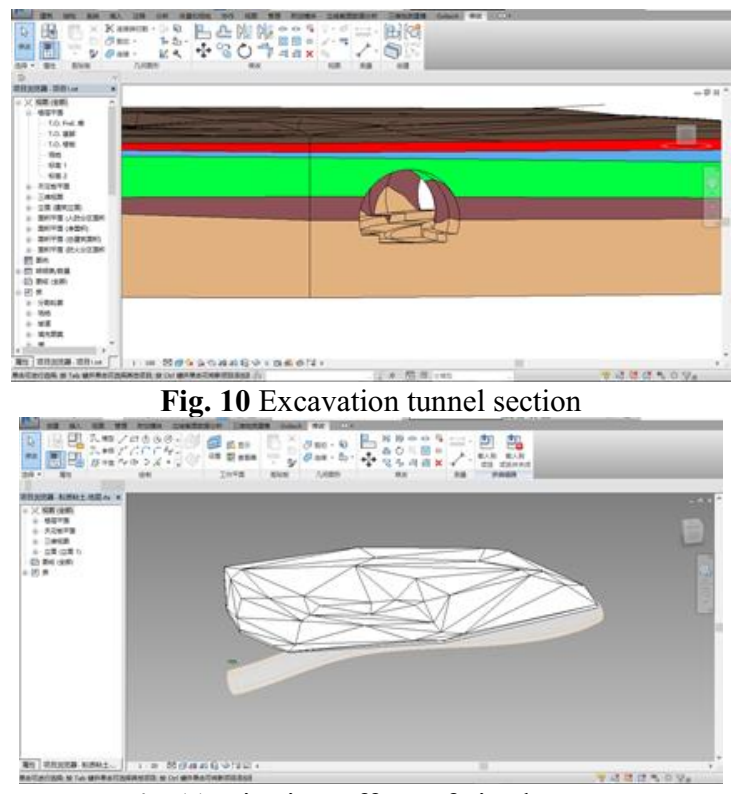

Fig.11 Digging effect of single stratum

\section{Conclusion}

The increasing maturity of BIM technology provides new targets and new ideas for geotechnical engineering survey and design methods. The three-dimensional geological information display technology based on BIM will provide decision makers with more intuitive decision-making basis and provide a design platform for cooperation and cooperation of various professions. This article relies on the Lizheng geotechnical survey data, based on the Revit secondary development technology to complete the preparation of virtual drilling and 3D geological modeling plug-in, which laid the foundation for the application of 3D geological models in actual engineering.

\section{Acknowledgements}

This work has been supported by the Natural Science Foundation of Hebei Province of China (Youth Program) under Grants \#E2020208071 and the Natural Science Foundation of Hebei Province of China (Grants \# E2019208159).

\section{References}

1. Lin Bingxian, Zhou Liangchen, Lu Guonian. Construction method of three-dimensional geological body model controlled by virtual drilling [J]. Journal of Earth Information Science, (2013), 15(05). 672-679.

2. Cai Guodong, Mao Weichen, Xie Chun, Xu Jie. Research on the application of Revit three-dimensional formation information model rapid modeling method [C]. Engineering Survey Branch of Chinese Architectural Society. Proceedings of the 15th National Conference on Engineering Geophysical and Geotechnical Testing. Engineering Survey Branch of China Architectural Society: Engineering Survey Branch of China Architectural Society, (2017):62-68.

3. Peng Jianlin. Three-dimensional geological modeling based on BIM-Taking a project in Wuzhifeng, Zhangjiang New District, Ganzhou as an example[J]. Fujian Construction, (2020)(03):118-121.

4. Chu Shili, Xia Mianli, Feng Mingming, Jiang Hualong, Ying Sheng. Research on Creation Method of Geotechnical Engineering Three-dimensional Geological Model Based on BIM technology[J]. Tunnel construction.2020,(39):152-157.

5. Li Yin, Yang Wunian, Yang Ronghao, Zeng Tao. Improvement of DEM interpolation algorithm based on moving surface fitting algorithm and weighted average algorithm $[\mathrm{J}]$. Surveying and Mapping,(2010), 33(04): 168-171.

6. Discipline Group of Surveying Adjustment, School of Surveying and Mapping, Wuhan University. Error Theory and Basis of Surveying Adjustment [M].Wuhan: Wuhan University Press, (2003). 